\title{
THE Design OF Digital Audio FILTER SYSTEM USEd IN TOMATIS Method StimULATION
}

Submitted: $8^{\text {th }}$ October 2018; accepted $7^{\text {th }}$ February 2019

\author{
Krzysztof Jóźwiak, Michał Bujacz, Aleksandra Królak
}

DOI: 10.14313/JAMRIS_1-2019/9

\begin{abstract}
:
The Tomatis Method is a rehabilitation technique used in psychology, the main aim of which is stimulating the cochlea in the inner ear by filtered air-conducted and bone-conducted sounds. The system of electronic filters and amplifiers used for this therapy is called the Electronic Ear. Commonly, it is a commercial analog device that is expensive and after a few years its functionality declines. In this paper, we propose a digital Electronic Ear system using an STM32F4 family micro-controller and ADC/ $D A C$ integrated circuits. The design of the digital sound filters allows to adjust more parameters and overcomes some of the constraints of analog systems. In this paper, we provide a short review of the Tomatis Method, the main functions of the Electronic Ear and we describe the designed system with comparison measurements to the analog original.
\end{abstract}

Keywords: Sound Filtering, Digital Signal Processing, Shelving Filter, FIR Filter, Electronic Ear Stimulator, Tomatis Method

\section{Introduction}

Music therapy has numerous applications in psychology and rehabilitation. It can be used to help patients manage stress, concentration or depression, even if in some cases these methods do not give significantly stronger effects, when they are compared with a control placebo group [1], [2], [3], [4].

The Tomatis Method (TM) has been shown to help people with various psychological disorders (autism, dyslexia, ADD and more) [10], [11], [12], [13], [14], [15], [16], [17], [18], [20] though in some controlled trials the results were also inconclusive [8], [19]. It uses music, but it is not purely music therapy, as it is based on brain and inner ear stimulation by selective sound filtering. The electronic device that implements the filter system for TM is called an Electronic Ear (EE). Most EEs that are commonly used are analog systems, that have their own constraints and high prices. We propose a new, digital Electronic Ear (DEE) system designed for cheaper implementation of TM and its further studies.

\section{Tomatis Audio-Psycho-Phonology}

Alfred Tomatis (1919-2001) was a professor, laryngologist and otorhinolaryngology specialist. He formulated the Tomatis Laws, which cover the theory behind the relationship of hearing and speaking. He observed that problems with singing or speaking in some particular spectrum are connected with difficulties with listening in the same spectrum and proposed that the improvement of vocal and voice range can be provided by opening the hearing ability to a specific frequency spectrum. This principle is called the Tomatis effect. He also emphasized the difference between listening and hearing - hearing is the ability to combine listening to a voice and focusing the attention on it. The main aim of psychological therapies based on Tomatis method was to improve not only the listening, but also the hearing ability. Over the years his ideas were implemented in other forms of sound therapy and a generic Electronic Ear tool was developed.

The Electronic Ear is based on the concept of stimulating the basilar membrane (BM) in the cochlea in two ways - by air and bone conduction. Air conduction is based on the typical ear pathway, with sound vibrations picked up by the eardrum, transferred by the ossicles to the oval window of the cochlea. Bone conduction is the perception of vibrations travelling through the bone and stimulating the BM either through pressure changes in the cochlear fluid or vibrations of the cochlear wall [5]. Bone conducted sounds reach the brain faster due to the higher density of the medium; however, large part of the acoustic signal is reflected due to the air-bone impedance mismatch. That's why bone conduction mostly favors our own voice. The conclusion drawn by Tomatis from this observation was that air conduction is used to "communicate with the outer world" and bone conduction is used to "listen to oneself". [6], [7].

The next feature that is important for TM is human earedness, i.e. which ear is dominant in the hearing process. Left ear dominance is problematic, because the left ear has the longer path to the left hemisphere. The delay between left and right ear is about 0,4-0,9 ms. People, who are left-eared have more frequent listening problems that can cause various psychological disorders, such as dyslexia [6]. One of the main aims of Tomatis Rehabilitation is to increase the role of the right ear for the people who are left-eared. For this reason there are separate delays and adjustable volume levels for left and right channels. 
In the beginning of the Tomatis rehabilitation a patient is tested for his audio attention capabilities by testing the lowest volume level of sound frequency that the patient can detect. The process is similar to a typical audiogram; however, it is performed four times: for air and bone conduction for the left and the right ear. The ideal shape is smooth, without sharp changes and with a peak in the range of $1-3 \mathrm{kHz}$. The bone conduction line should be exactly $5 \mathrm{~dB}$ lower than the air conduction.

Fig. 1 shows the flow chart of the EE. It is an audio system, the main function of which is amplifying, gating and filtering sound going to the three output channels - air left, air right and bone conduction. Gating is the process of changing the filter type depending on signal level. Below a certain amplitude level the C1 filter is on and above a certain level the filter is switched to C2 filter. The sudden change gives an impulse that theoretically stimulates the brain. The $\mathrm{C} 1$ and $\mathrm{C} 2$ filters are shelving filters with $1 \mathrm{kHz}$ cross-band frequency. The boost/attenuation level is adjusted by the specialist administering the TM therapy and can be set from -5 to $5 \mathrm{~dB}$. The EE also contains a second filter - a High Pass filter with an adjustable cut-off frequency. Its supposed function is to imitate the medium of a mother's womb - the environment, where the hearing system of a fetus was developing. In some cases there is a need to increase or decrease a role of bone conduction in comparison to air conduction. For this reason there is an adjustable delay between the air and bone channel called precession.

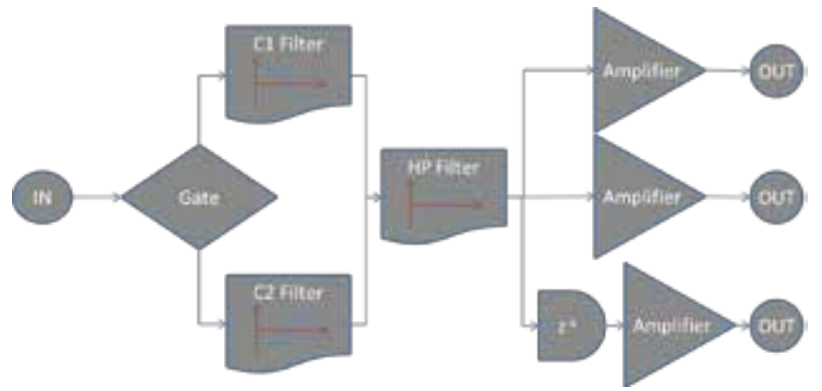

Fig. 1. Flow chart of the Electronic Ear (EE). The Gate depending on the signal level diverts it to the shelving filters C1 or C2. The HP filter cuts off unpleasant low frequencies. The Delay allows to the air and bone conducted sounds to arrive at different times and the Amplifiers control the level of the three outputs

TM is used for many purposes. One of the popular aims is in therapy of autistic children. According to Tomatis theory autism is connected with problems with listening to the external world - and it is true that in most autistic children bone conduction sensitivity is higher than air conduction. The therapy's aim is to lower this sensitivity in order to open the child up to communication with others. There were a number of studies carried out to verify the effectiveness of this rehabilitation. One of the most controversial was Brief Report: The Effects of Tomatis Sound Therapy on Language in Children with Autism [8]. It revealed that there was no clear difference between the effects on a control group and an experimental group put under
TM rehabilitation. For response to this article there were two follow-up studies - Sound Therapy: an Experimental Study with Autistic Children [10] and Response to "Brief Report: The Effects of Tomatis Sound Therapy on Language in Children with Autism [9]. They showed the opposite view and pointed out missteps in the previous research. These two and another one [11] show a significant improvement after the TM rehabilitation measured in the Children's Autism Rating Scale (CARS) or in Gilliam Autism Rating Scale (GARS).

An overview of over 30 TM studies by Gerritsen showed that the majority of studies demonstrated positive effects of TM therapy. Some of the better documented TM treatments include:

- reduction of the Attention Deficit Disorder (ADD) by, similarly to autism, increasing the focus on air conducted sounds versus bone conduction [6], [12], [13]

- treating dyslexia [6],[13],[20] (that is theoretically connected with left-earedness) by increasing the sensitivity of the right ear

- supporting language learning [14], by setting the $\mathrm{C} 1 / \mathrm{C} 2$ filters to frequencies most commonly appearing in a given language.

TM rehabilitation has also been successfully used for:

- Epilepsy - visible effect in more than $50 \%$ of tested patients [15]

- Cerebral palsy - significant improvement [16]

- Stuttering and hoarseness [13]

- Emotional problems including depression - high effect, compared with a control group [17]

- Music skills improvement - results are divided [18], [19].

Most of tests have shown a positive effect of TM, but there are also those, that did not report any differences when compared to a placebo treatment. We need to consider that negative results can stem from inappropriateness in investigation methods or in the way the EE device was used [9]. This shows that the TM requires further rigorous studies.

\section{Electronic Ear Stimulator}

In terms of the electronic design main functions of Electronic Ear are:

1. Converting audio signal to digital data (ADC)

2. Pre-processing

3. Gating

4. Filtering

5. Digital-Analog converting (DAC)

6. Amplifying

The device can be divided into four main modules: audio input, audio output, micro-controller and power supply. Three voltage levels are needed:

- 5V -external power supply. It is used as an input for the step-down converter, LCD power supply and as an analog power supply for the input section. Ferrite beads are used between the analog and digital sections. A transformer power source is used in order to decrease the distortions in the low frequency audio signals. 
- $3.3 \mathrm{~V}$ - it is given by Low Dropout Positive Regulator TC2117 from MicroChip. It is used for digital power supply for $\mu \mathrm{C}$ and for the digital part (serial communication interfaces I2S and I2C) of the ADC/ DAC converters.

- $2.5 \mathrm{~V}-$ it is necessary for the output analog power supply and it is provided by LP2985-N - Low dropout Linear regulator from Texas Instruments. It can provide $150 \mathrm{~mA}$ current for the normal work conditions, that is enough for the purpose of this project.

The maximum resolution for ADC and DAC converters built into the STM32F4 family controllers is 12-bits. Since this is insufficient for this project, external 24-bit stand-alone integrated circuits are used. For input a PCM4201 from Texas Instruments and for output CS432L22 from Cirrus Logic are used. In both cases communication between the audio converter and $\mu \mathrm{C}$ is provided by an I2S interface, the audio data word length is 24 bits and is Left-Justified in the frame. CS43L22 supports I2S and it is working in the slave mode by being connected to four pins:

- SCLK - clock signal for the serial interface

- LRCK - clock signal determining which channel is currently sent by SDO line. Its frequency is equal to the sampling frequency.

- SDO - audio data digital signal

- MCLK - MCLK line is necessary for proper work of CS43L21 since it serves as a clock source for the delta-sigma modulators.

The input converter communication protocol is not completely compatible with I2S. PCM4201 has its own transmission interface that has 3 communication ports - BCK (equivalent to SCLK), DATA (equivalent to SDO), FSYNC (equivalent to LRCK) and one system clock port, that is necessary for proper work. The serial interface is very similar to I2S and after a few modifications it can work in high performance mode communicating in master mode. The main difference is that the system clock needs to be 512 times larger than the sampling frequency (256 times larger in I2S mode). To get that result an extra I2S interface is used with a 2 times larger sampling frequency and the MCLK pin connected to the system clock pin in the audio converter. That allows a clean transmission without errors.

The STM32F4 microcontroller works in the I2S master mode for the communication with the output DAC converter. Since the input ADC converter has its own protocol, it has to work in the master mode and $\mu \mathrm{C}$ works in the slave mode. For the STM32F401 microcontrollers a frame synchronization error occurs when the I2S interface works in the slave mode. In order to solve this problem, after the serial interface initialization and before communication start the LRCK pin has to be set high internally from the device.

CS43L22 has two serial interfaces and except of I2S for data transmission it also has an I2C interface for control. It is needed to initialize the device and set the work conditions. It allows also to regulate all functions of the chip during work, for example - volume, that is continuously sent to the control register in the main loop of program.
The CS43L22 is a DAC converter, but also a headphone amplifier. It allows to prevent from using two independent chips (one for converting, one for amplifying) and minimalizes space on the PCB board. In the project there are two output channels - air and bone channel. In order to support three channels (that are required for the EE) there is used an external adapter that splits the air channel to left and right channels with a build-in balance regulator. The headphone amplifier can provide $88 \mathrm{~mW}$ power for $16 \Omega$ output. The air channel lines of the measured headphones have $320 \Omega$ impedance, so the parallel connection impedance is equal to $160 \Omega$. The bone conduction vibrator has $32 \Omega$ impedance. In those conditions the $150 \mathrm{~mA}$ power supply used in this project is sufficient and all of the three output channels are supplied.

For digital signal processing the STM32F407VGT6 microcontroller from STMicroelectronics is used. It has a CPU frequency of up to $168 \mathrm{MHz}, 1$ Mbyte of flash memory and 196 Kbytes of static RAM memory. It provides three I2C and two I2S serial communication interfaces. The $144 \mathrm{MHz}$ clock is used to minimize the error of timer frequency to less than the sampling frequency and I2S clock signal frequency (the error is equal to $0.017 \%$ ). Data is stored in a float buffer. Its size is set in order to provide maximum 500 milliseconds delay, which for $48 \mathrm{kHz}$ frequency sample gives (24000 + maximum filter order) size. The SRAM memory has enough space to store all data needed, so the flash memory is not used in this project.

User interface gives possibility to adjust the main parameters of the EE - C1, C2 levels value, filters frequencies, precession and volume and the advanced options as gate upper level and four options for filter order. Changing filter order affects its selectivity and gate level includes the $\mathrm{C} 1 / \mathrm{C} 2$ filters switching rate. Both parameters determine the intensity of stimulation. Volume control is provided by sending a proper value to the register of amplifier, the same level for air conduction and bone conduction channel. The gain level difference between both channels has to be added in the program function after uploading the ADC value before signal processing. It is necessary, because the gate has to switch these two channels separately depending on bone conduction gain level and precession value.

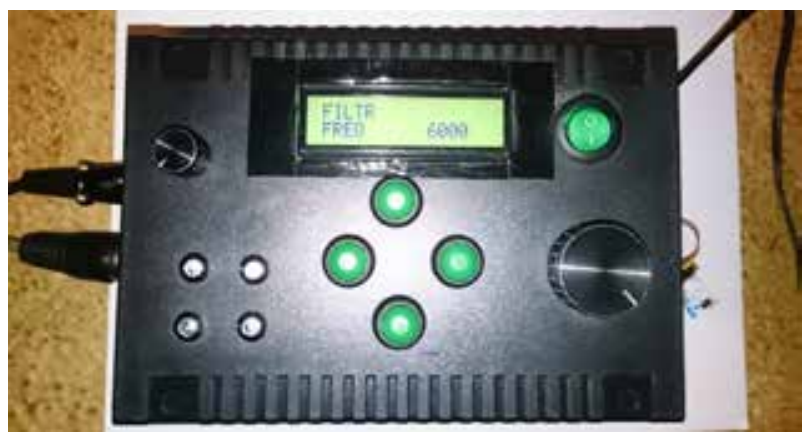

Fig. 2. Picture of the designed device

Fig. 2. shows the picture of the designed equipment. The prototype of the device is made with a plastic case. It uses an LCD display with HD44780 
driver to communicate with the user. The four buttons and an incremental encoder are used for navigating a menu and adjusting all of the device parameters in an easy way. The device contains four LED diodes to inform which filter is currently used - C1 or C2 filter for air and bone conduction channel. The potentiometer on the left side of the case is used for adjusting the proper pre-amplifier gain. This is an effective way to increase the quality of the sound by making use of all the 24 bits of the ADC converter.

In this project two types of filters are required simple High-Pass \& Low-Pass filters and a shelving filter for the $\mathrm{C} 1$ and $\mathrm{C} 2$ filtering. The analog filters were commonly implemented using the original Electronic Ear. The FIR (Finite Impulse Response) filters were chosen for this project. Digital FIR filters are not able to be derived from analog filters, because the analog ones always give an infinite impulse response. However, the main goal of the project was to design the digital system without analog filters disadvantages instead of simply copying the original. The FIR filters are inherently stable and have a linear phase being designed with a proper method. The Discrete Fourier transform (DFT) is used to perform linear filtering. The filter impulse response is approximated to function $\sin (x) / x$.

The filter orders in range of 51 to 101 are used for this project. The designing method with only the odd orders was chosen. Every filter is designed as FIR filter using the window method. The window that was chosen for this purpose is modified Tukey window (tapered cosine window). It is combination of a rectangular window and a Hamming window with an $\alpha$ coefficient that determines what part of the window should be flat. It makes cosine lobe of width $\alpha / 2 * N$ ( $N$ is filter order) and rectangular window of width $(1-\alpha / 2) * N$ at the center of filter function. With $\alpha=0.8$ it gives the best results - sufficient filter selectivity providing expected filter band levels even for low cross-frequency $(500 \mathrm{~Hz}$ is a minimum used value) and low ripple frequency response.

To obtain a C1 \& C2 two-level shelving filter it was necessary to use a parallel connection of filters - the multiplying filters coefficients presented in $\mathrm{Z}$ transform. Two simple filters are multiplied - one for frequencies below the cross-frequency (LP filter for a left part of characteristic) and one for frequency range above the cross-frequency (HP filter). The cross-frequency is set where the characteristic should reach the $0 \mathrm{~dB}$ line. For that reason there is a need to modify one frequency (adjusted by the user), by a coefficient dependent on filter order and C1 \& C2 levels.

The big challenge was to appropriately design the filter in case when both shelving filter levels (lower and upper than cross-frequency) have the same sign. Normally it should not cross the zero decibels line. But measurements of the original EE show, that with this case it should be artificially modified in order to give $0 \mathrm{~dB}$ gain at the cross-frequency point. A cascade connection of two filters with similar cut-off frequency was used, but modified regarding to the purpose. For the positive shelving filter levels there is a need to decrease the gain near the cross-frequency (frequen- cies of filters diverge) and for negative it should be increased (frequencies converge). Then, obtained filter is serially connected (adding filter coefficients in the Z-transform domain) with the normal shelving filter, that is computed by the usual method.

\section{Results}

In this section the frequency-responses of C1 \& C2 shelving filters in the designed DEE are shown and compared with those measured for the original, analog EE device.

All measurements were made for input signal frequency in range of $20 \mathrm{~Hz}$ to $16 \mathrm{kHz}$ with variable steps depending on the cross-frequency value. The output signal voltage levels on the $\mathrm{Y}$ axis are presented as relative values to All-Pass filter characteristic ( $\mathrm{C} 1$ and $\mathrm{C} 2$ equal to $0 \mathrm{~dB}$ ).

Fig. 3 and Fig. 4 show frequency-responses for 101-order digital filters for designed device. As it can be seen, passband levels have correct values, remain stable and C1 \& C2 filter lines cross near the $0 \mathrm{~dB}$ level. In the first case the $500 \mathrm{~Hz}$ (the minimal needed value for this project) and in the second $1 \mathrm{kHz}$ (frequency of analog EE) cross-band frequency is set. In Fig. 4 the filter with two positive levels is shown to provide appropriate gain modification. The characteristic fall into $0 \mathrm{~dB}$ value near cross-frequency and then return to a stable level.

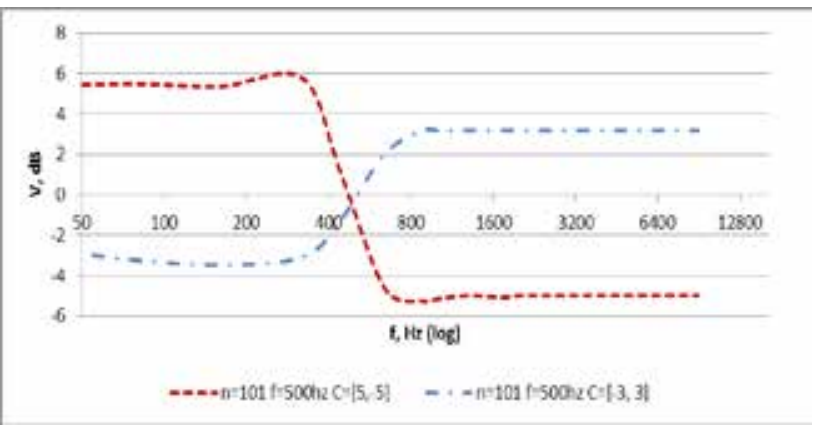

Fig. 3. Frequency-response for $n=101, f=500 \mathrm{~Hz}$ and passband levels set

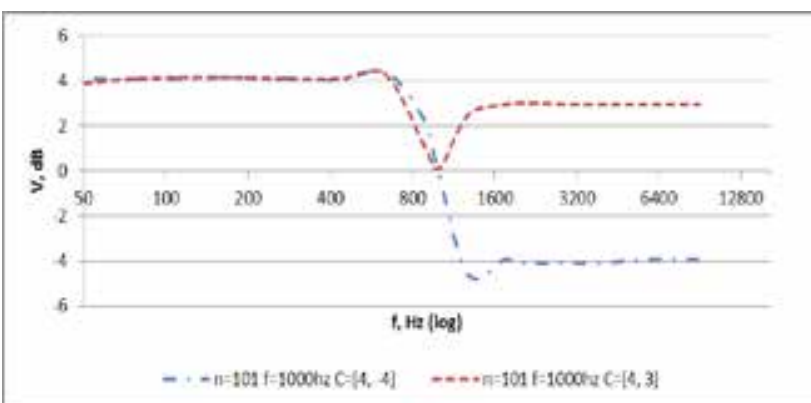

Fig. 4. Frequency-response for $n=101, f=1000 \mathrm{~Hz}$ and passband levels set

In the Figs 5 and 6 frequency responses measured for the DEE and analog EE are shown for $1 \mathrm{kHz}$ crossband frequency and $[ \pm 5, \pm 5]$ and $[ \pm 5, \pm 1]$ levels respectively. All filters of the analog EE are expected to 
have $1 \mathrm{kHz}$ zero-cross frequency with a slope of $6 \mathrm{~dB} /$ octave. The unstable and exceeded passband levels during the whole frequency bandwidth for analog devices can be seen. In the Fig. 6 one can notice the ringing artifacts for the bandwidth above $1 \mathrm{kHz}$. The cross-point for analog device also has an incorrect value i.e. $850 \mathrm{~Hz}$ for first case and $865 \mathrm{~Hz}$ for the second case.

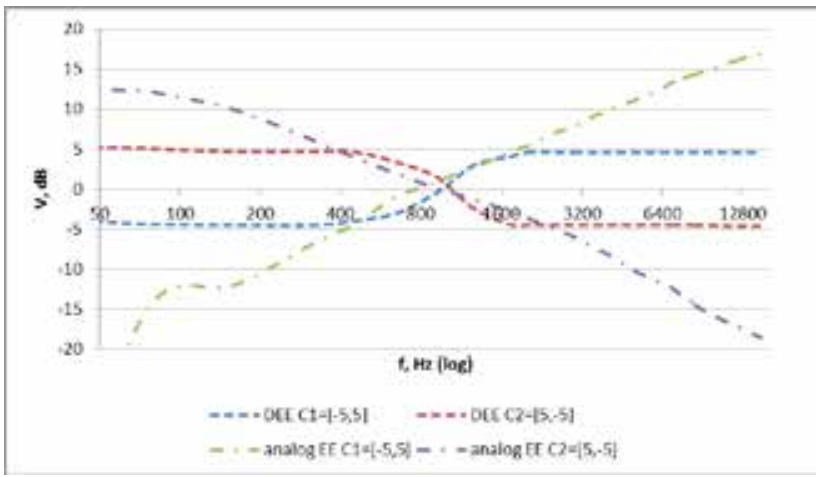

Fig. 5. Frequency-response of $\mathrm{C} 1 / \mathrm{C} 2$ filters measured for digital and analog device. Theoretically, the analog EE's pass bands should be flat, but the measurements show they clearly have a linear drop-off

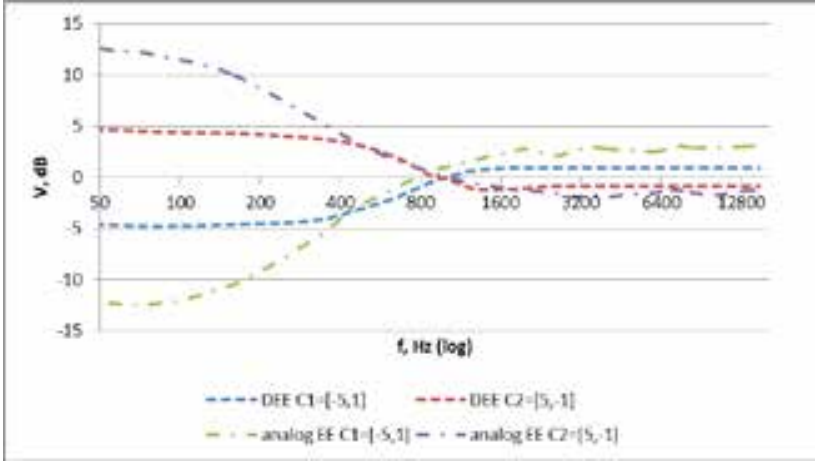

Fig. 6. Frequency-response of $\mathrm{C} 1 / \mathrm{C} 2$ filters measured for the proposed digital and the original analog TM device

\section{Conclusion}

The DEE, which was the topic of this paper was designed properly and it provides all the required functions. It services 2 channels, that with external splitting adapter allow to support three channels. If the right/left channel delay was needed, there would be a possibility to use second, same DAC integrated device for extra output channels. The DEE uses 24-bit audio data with $48 \mathrm{kHz}$ sampling frequency. The filters, that it includes, give the desired effects the shelving filter levels and cross-frequency value are as expected. It monitors changes at air and bone channels and gates them to $\mathrm{C} 1$ or $\mathrm{C} 2$ filters depending on the signal level and precession value.

Compared to the analog EE, the designed device has all the same functions, but it has also extra- features allowing to remove some previous constraints. First it is a digital system, so the filter parameters are stable and do not depend on the duration of operation or component aging. In Fig. 5 and 6 there is visible effect of this occurrence for the analog devicethe filter levels are different than expected and are unstable. Another advantage is that the cost of production of the DEE (less than $200 €$ ) is much lower than the analog TM device (approximately $10000 €$ ). The fact that filters in the designed system are digital also gives an opportunity to develop it in the future, change the parameters or to upgrade it depending on the requirements.

The next advantage is that digitalization allows more parameters to be adjusted by the user. The main and very important difference is the possibility to set the cross-frequency of the shelving filter. In analog EE $\mathrm{C} 1$ and $\mathrm{C} 2$ filters have always the same cross-frequency and it is $1 \mathrm{kHz}$. It has also only one type of filter selectivity. In the designed device there are four cases for filter order. It allows to change the intensity of stimulation and pick the best one for the individual patient. The changeable filter frequency goes with opportunity to modify frequency band, that impacts the most to the listener.

To sum up, a modernized digital version of the device used for the rehabilitation by the Tomatis method was designed and tested. Its performance was measured to be the same or better than the original equipment, while allowing much more control over the functionality.

\section{AUTHOR}

Krzysztof Jóźwiak* - Lodz University of Technology, Institute of Electronics, ul. Wólczańska 211/215, 90-924 Łódź, Poland, email: kjozwiak@dmcs.pl.

Michał Bujacz - Lodz University of Technology, Institute of Electronics, ul. Wólczańska 211/215, 90-924 Łódź, Poland, email: michal.bujacz@p.lodz.pl.

Aleksandra Królak - Lodz University of Technology, Institute of Electronics, ul. Wólczańska 211/215, 90-924 Łódź, Poland, email: aleksandra.krolak@p.lodz.pl.

*Corresponding author

\section{REFERENCES}

[1] L. Hohmann, J. Bradt, T. Stegemann, and S. Koelsch, "Effects of music therapy and music-based interventions in the treatment of substance use disorders: A systematic review", PLOS $O N E$, vol. 12 , no. 11,2017

DOI: 10.1371 /journal.pone.0187363.

[2] M. Bodner, R. P. Turner, J. Schwacke, C. Bowers, and C. Norment, "Reduction of Seizure Occurrence from Exposure to Auditory Stimulation in Individuals with Neurological Handicaps: A Randomized Controlled Trial", PLOS ONE, vol. 7, no. 10, 2012 DOI: $10.1371 /$ journal.pone.0045303.

[3] M. Bucur and A.-L. Marian, "The impact of the Mozart effect on creativity: myth or reality", Creativity \& Human Development, 2016, www.creativityjournal.net/newsevents / item/298-the-mozart-effect-and-creativity. 
[4] L.-C. Lin, W.-T. Lee, H.-C. Wu, C.-L. Tsai, R.-C. Wei, H.-K. Mok, C.-F. Weng, M.-w. Lee, and R.-C. Yang, "The long-term effect of listening to Mozart K.448 decreases epileptiform discharges in children with epilepsy", Epilepsy \& Behavior, vol. 21, no. $4,2011,420-424$

DOI: 10.1016/j.yebeh.2011.05.015.

[5] P. Henry and T. Letowski, Bone conduction: Anatomy, physiology, and communication, Army Research Laboratory, 2007.

[6] P. Sollier, Listening for wellness: An Introduction to the Tomatis Method, The Mozart Center Press, 2005.

[7] N. Doidge, The brain's way of healing: remarkable discoveries and recoveries from the frontiers of neuroplasticity, Viking: New York, 2015.

[8] B. A. Corbett, K. Shickman, and E. Ferrer, "Brief Report: The Effects of Tomatis Sound Therapy on Language in Children with Autism", Journal of Autism and Developmental Disorders, vol. 38, no. 3, 2008, 562-566 DOI: $10.1007 /$ s10803-007-0413-1.

[9] J. Gerritsen, "Response to "Brief Report: The Effects of Tomatis Sound Therapy on Language in Children with Autism", July 3, 2007, Journal of Autism and Developmental Disorders", Journal of Autism and Developmental Disorders, vol. 38, no.3, 2008, 567-567

DOI: $10.1007 / \mathrm{s} 10803-007-0471-4$.

[10] M. AbediKoupaei, K. Poushaneh, A. Z. Mohammadi and N. Siampour, "Sound Therapy: An Experimental Study with Autistic Children", Procedia - Social and Behavioral Sciences, vol. 84, 2013, 626-630 DOI: $10.1016 /$ j.sbspro.2013.06.615.

[11] J. M. Neysmith-Roy, "The Tomatis Method with Severely Autistic Boys: Individual Case Studies of Behavioral Changes", South African Journal of Psychology, vol. 31, no. 1, 2001, 19-28 DOI: $10.1177 / 008124630103100105$.

[12] L. Sacarin, "Early Effects of the Tomatis Listening Method in Children with Attention Deficit", Dissertations \& Theses, 2013, 44, https://aura. antioch.edu/etds/44.

[13] J. Gerritsen, "A Review of research done on Tomatis Auditory Stimulation", 2009.

[14] I.-M. du Toit, W. F. du Plessis, and D. K. Kirsten, "Tomatis Method Stimulation: Effects on Student Educational Interpreters", Journal of Psychology in Africa, vol. 21, no. 2, 2011, 257-265 DOI: $10.1080 / 14330237.2011 .10820454$.

[15] G. Coppola, A. Toro, F. F. Operto, G. Ferrarioli, S. Pisano, A. Viggiano, and A. Verrotti, "Mozart's music in children with drug-refractory epileptic encephalopathies", Epilepsy \& Behavior, vol. 50, 2015, 18-22 DOI: 10.1016/j.yebeh.2015.05.038.

[16] I. Przybek-Czuchrowska, E. Mojs, and E. UrnaBzdęga, "Opis przypadku dziecka z organicznym uszkodzeniem w obrębie ośrodkowego układu nerwowego leczonego metodą treningu słuchowego Tomatisa (Case study of a child with or- ganic damage within the central nervous system treated with the Tomatis method)", Neuropsychiatria i Neuropsychologia / Neuropsychiatry and Neuropsychology, vol. 10, no. 1, 2015, 40-45 (in Polish).

[17] J. O. Coetzee, The effect of the Tomatis Method on depressed young adults, 2001.

[18] W. du Plessis, S. Burger, M. Munro, D. Wissing, and W. Nel, "Multimodal Enhancement of Culturally Diverse, Young Adult Musicians: A Pilot Study Involving the Tomatis Method", South African Journal of Psychology, vol. 31, no. 3, 2001, 35-42 DOI: $10.1177 / 008124630103100305$.

[19] I. Vercueil, H. Taljaard, and W. d. Plessis, "The effect of the Tomatis Method on the psychological well-being and piano performance of student pianists: An exploratory study", South African Music Studies, vol. 31, no. 1, 2011, 129-158.

[20] T. Gilmor, "The Efficacy of the Tomatis Method for Children with Learning and Communication Disorders: A Meta-Analysis", International Journal of Listening, vol. 13, no. 1, 1999, 12-23 DOI: $10.1080 / 10904018.1999 .10499024$. 